\title{
NEEDS for LHC experiment planning from results of very high energy cosmic ray Investigations (NEEDS-2)
}

\author{
A.A. Petrukhin ${ }^{\mathrm{a}}$ \\ National Research Nuclear University MEPhI (Moscow Engineering Physics Institute), Russia
}

\begin{abstract}
ISVHECRI, a special NEEDS workshop was held to discuss future LHC data required for interpretation of cosmic ray experiments. Now, when the main task of LHC is solved - the Higgs boson is discovered - the question "What will be the next?" is very actual. In this paper the results of cosmic ray experiments at LHC energies are considered. Their possible explanation in the frame of a new model of production of quark-gluon matter blobs is discussed. The necessity to pass in LHC experiments from investigations of $p p$-interactions to investigations of nucleus-nucleus interactions is underlined since cosmic rays consist mainly of nuclei $(\approx 60 \%)$ which interact with nuclei of air. But namely in these nucleus-nucleus interactions many unusual results were obtained in cosmic ray investigations. Corresponding tasks for future LHC experiments are proposed.
\end{abstract}

\section{Introduction}

A correspondence between the intervals of energy $10^{15}$ $10^{18} \mathrm{eV}$ in cosmic ray experiments and energy of protonproton, proton-nucleus and nucleus-nucleus beams in LHC (several $\mathrm{TeV}$ per nucleon) is well known. But a liaison between experiments (and between experimentalists) in cosmic rays and at accelerators is relatively weak. There are at least two reasons for that.

The first are the different purposes, tasks and correspondingly methods of investigations. For the accelerator physicists the main purpose is the search for new particles: Higgs boson, supersymmetric particles, etc. For that, large transferred momenta and correspondingly large angles of particle fly-out to the direction of beams are the most acceptable. In cosmic ray experiments, only results of primary particle interactions with nuclei of atoms of the atmosphere can be investigated, and neither energy nor type of particle are known. Detectors at the Earth's surface register results not only of primary interaction but of multiple secondary interactions, too. Taking into account the steep dependence of cosmic ray intensity on the energy as $E^{-2.7}$, the main contribution in many detected events give secondary particles with largest energies which fly at very small angles. In colliding experiments, particles at such angles cannot be practically measured.

The second reason is a very skeptic attitude of the accelerator community to results of cosmic rays experiments due to their drawbacks (unknown type and energy of primary particles) and poor statistics. Furthermore, before LHC, the energies of previous colliders Tevatron and RHIC were insufficient for comparison with $\mathrm{PeV}$ energy region in cosmic ray experiments.

\footnotetext{
a e-mail: aapetrukhin@mephi.ru
}

The beginning of LHC experiments changed the situation and required joining of cosmic ray and accelerator physicists. The first step was done in April 2002 when in Karlsruhe Forschungszentrum the first NEEDS Workshop (Needs from Accelerator Experiments for the Understanding of High-Energy Extensive Air Showers) was organized [1]. The second NEEDS Workshop was held during $12^{\text {th }}$ ISVHECRI in 2002 [2]. As a result of this Workshop, the following questions were formulated [3]. Since the most abundant hadrons in EAS are charged pions, their interactions with nuclei of nitrogen and oxygen were named as a primary importance. The second priority obtained interactions of protons, neutrons and kaons with these nuclei. And only the fourth priority got interactions of more heavy nuclei $(\mathrm{C}, \mathrm{O}, \mathrm{Fe})$ with nuclei of atoms of the atmosphere.

What is the situation now, twelve years later? Of course, the discovery of Higgs boson is the outstanding achievement in modern physics but its influence on cosmic ray investigations is negligible. The results obtained in $p p$-interactions confirm the basic predictions of models which were developed on the basis of cosmic ray experimental data. Some discrepancies between experimental data and theoretical calculations can be easily eliminated by fine tuning of theoretical models. But practically no information which can help to understand and explain the numerous unusual results observed in cosmic rays was obtained.

The main reason is related with particles which are investigated at colliders and in cosmic rays. LHC experiments are directed mainly at $p p$-interactions. In cosmic rays most particles (60\%) are nuclei, and only $40 \%$ are protons. And results of practically all experiments at very high energies (above the knee) show an increase of the number of nuclei compared to protons. It means that in cosmic ray experiments the most interactions are nucleusnucleus interactions, and namely in these interactions

This is an Open Access article distributed under the terms of the Creative Commons Attribution License 4.0, which permits unrestricted use, distribution, and reproduction in any medium, provided the original work is properly cited. 
various unusual events were observed. Therefore now the question can be turned over: What consequences for future LHC experiment planning follow from results of cosmic ray experiments?

\section{What are the evidences from cosmic ray experiments?}

The first phenomenon which can be considered as evidence of a change of interaction model was the observation of a change in the dependence of the total number of charged particles on energy in EAS investigations. Such a possibility (but as a likely option) was considered in the first paper about the knee observation [4], but very soon the choice in favor of cosmophysical explanation of the knee was made. Today most cosmic ray physicists consider the knee as a proof of cosmic ray origin in the Galaxy, the size and magnetic field of which determine the knee energy. Only in recent years were serious arguments in favour of nuclear physical approach discussed [5].

The second phenomena were several types of unusual events in hadron-hadron interactions: halo, alignment, Centauros, penetrating cascades, long-flying component, etc. These events could not be explained by cosmophysical reasons, and various proposals of new interaction models began to be discussed, but over the years no model which could explain a full set of unusual events was proposed. In paper [6], an attempt to combine the reasons of the appearance of these unusual events and the knee in the frame of a single approach was undertaken.

The last group of phenomena, which cannot be explained in the frame of existing models, are related with muon component (so called "muon puzzle" [7]). This puzzle includes two types of experimental results: an excess of muon bundles compared to calculations even for pure iron cosmic ray composition for any existing model of interaction, and an excess of very high energy muons (more than $100 \mathrm{TeV}$ ) compared to calculations of inclusive muon energy spectrum. For an explanation of the first excess (muons in EAS) it is possible, in principle, to develop a corresponding model of interaction, though it will not be a simple task. An explanation of the second excess (a change of the muon energy spectrum behavior at energy above $100 \mathrm{TeV}$ ) is a more complex task. For that, the decays of any heavy particles are required.

\section{What do we need to explain all unusual data?}

It is necessary to underline that an approach which can explain all observed data (not some part of them) is required. For example, the change of the muon energy spectrum at energy about $100 \mathrm{TeV}$ can be explained by a sharp increase of the cross section of charmed particle production. But these particles will not be useful for explaining other observed phenomena.

So, we need a model of hadron interactions which gives:

1. Threshold behavior (unusual events appear at several PeV only).
2. Large cross-section (to change EAS spectrum slope).

3. Large orbital momentum (alignment).

4. Large yield of VHE leptons (excess of VHE muons, muon bundles, penetrating cascades).

5. The change of EAS development and, as a consequence, increasing $N_{\mu} / N_{\mathrm{e}}$ ratio.

It is easy to fulfill the first condition by production of a new heavy particle (state of matter). If this object is connected with the appearance of the knee in cosmic ray energy spectrum, its mass must be $\sim \mathrm{TeV}$.

The second condition is the most difficult one and, at the first sight, it contradicts the first condition, since the geometric cross-section of heavy particle production

$$
\sigma \sim \pi \lambda^{2} \sim \pi / m^{2}
$$

and for $m \sim 1 \mathrm{TeV}, \sigma$ will be equal to $\sim 10^{-33} \mathrm{~cm}^{2}$. However, if we consider the production not of the particle but of some new state of matter, e.g. a blob of quark-gluon plasma (matter), the situation is changed [8]. In this case a transition from quark-quark (quark-gluon, gluon-gluon) interactions to collective interaction of many quarks and gluons is required. This directly leads to the appearance of a large cross-section:

$$
\sigma \sim \pi \lambda^{2} \rightarrow \pi R^{2}
$$

where $R$ is a size of a blob of quark-gluon matter, which mass and size cannot be less than mass and size of several nucleons.

The production of blobs of QGM provides also the first condition, since for QGM formation a high temperature (energy) is required. But to explain other observed phenomena a large value of orbital angular momentum is necessary.

In 2005, the theoretical paper was published [9], in which it was shown that in non-central ion-ion collisions a large orbital angular momentum must appear, and its value is proportional to the collision energy in the center-of-mass system. The further development of this approach showed that the value of this momentum can reach $\sim 10^{4}[10]$. This circumstance drastically changes the situation.

In usual consideration, quark-gluon matter produced in heavy nucleus interactions decays very quickly into secondary particles, and to separate them from particles generated directly in nucleus-nucleus collisions is practically impossible. A blob of a globally polarized QGM with a large orbital angular momentum can be considered as a usual (but very massive) resonance with a large centrifugal barrier. Its value

$$
V(L) \sim L^{2} / 2 m R^{2}
$$

will be large for light quarks but much less for top quarks. Although in interacting nuclei top-quarks are absent, the suppression of decays into light quarks gives time for their appearance. 


\section{How is the interaction changed in the frame of a new model?}

Firstly, simultaneous interactions of many quarks change the energy in the center-of-mass system

$$
\sqrt{s}=\sqrt{2 m_{N} E_{0}} \rightarrow \sqrt{2 m_{c} E_{0}},
$$

where $m_{N}$ - nucleon mass; $m_{c} \approx n m_{N}$ - compound mass of a part of target nucleus which together with a part of projectile nucleus is included in the blob of QGM. This change of $\sqrt{s}$ will have a big influence in interpreting various experiments (see below).

Secondly, decay of QGM blob into top-anti-top pair decreases $\sqrt{s}$ at energy about $700 \mathrm{GeV}$ or more (mass of two top-quarks is about $350 \mathrm{GeV}$, and flyout energy $\sim 350 \mathrm{GeV}$, too). As a result, the energy of secondary particles from such nucleus-nucleus interaction is decreased and further development of the cascade process is also changed. The life time of the top-quark is very small $\left(\sim 10^{-25} \mathrm{~s}\right)$ and it decays into $W$-boson and $b$-quark. $W$-bosons in their turn decay into leptons $(\approx 30 \%)$ and hadrons $(\approx 70 \%)$, in the first case charged leptons $(\mathrm{e}, \mu, \tau)$ and three types of neutrinos appear. In the center-of-mass system their energies will be about $40 \mathrm{GeV}$, but in cosmic ray interactions their energy will be above $100 \mathrm{TeV}$. In the decay of $W$-boson into hadrons (mainly pions, on average about 20), the total number of secondary pions is increased and correspondingly the number of muons is increased, too.

\section{What can explain the new model?}

Short answer: practically all unusual events and phenomena. With increasing primary cosmic ray energy, the initial energy of QGM blobs will be increased, and probability of their decays into top-quark pairs will also be increased. Therefore both muon puzzles (excess of muon bundles and excess of VHE muons) get their explanations. For example, the introduction in CORSIKA of the top-quark production by using PYTHYA explains the excess of VHE muons very well (Fig. 1). Such muons can explain penetrating cascades due to their consecutive interactions.

Production of top-quarks changes the development of EAS in the atmosphere (Fig. 2) due to increasing secondary particle multiplicity and appearance of a missing energy, since in EAS simulations VHE muons and neutrinos are not taken into account. The similar situation is in the LHC experiments. Well-known results of a sharp increase of multiplicity of secondary particles observed in lead-lead interactions [11] (Fig. 3) can be interpreted in the frame of a new model in the following way. In Fig. 3, the experimental points correspond to the centreof-mass energy $\sqrt{s_{N N}}=2.76 \mathrm{TeV}$, calculated for nucleon target mass. If one takes into account some compound mass, the positions of these points must be shifted to the right (Fig. 3). Taking into account that multiplicities in A-A-interactions cannot be less than in $p p$-interactions and the curve of its dependence on energy must not cross the curve for $p p$-interactions, it is possible to evaluate limiting number of nucleons included in QGM blobs.

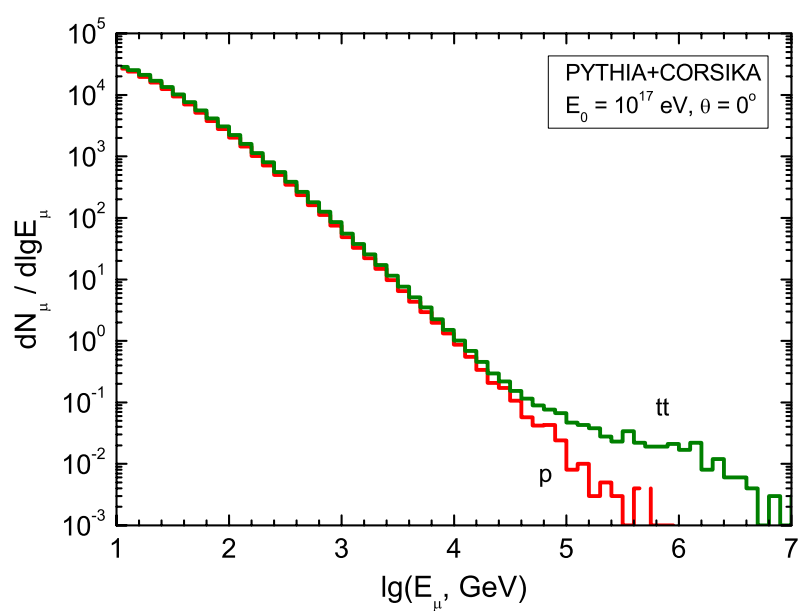

Figure 1. The inclusive muon energy spectrum simulated by means of CORSIKA taking into account t-quarks pair production according to PYTHYA.

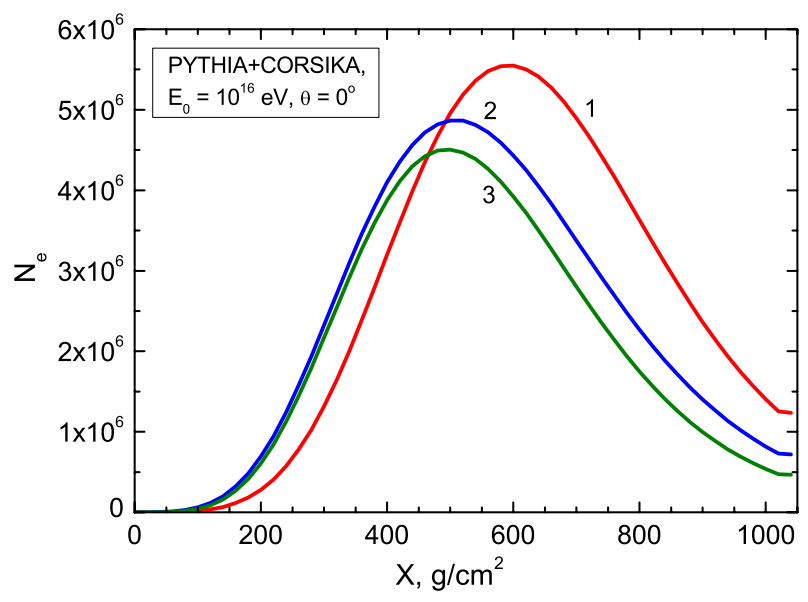

Figure 2. EAS cascade curves for various primary particles and different models of interaction: 1) proton and 2) iron with standard model, 3) proton with production of t-quarks.

The shifting of the experimental point from $\sqrt{s_{N N}}=$ $3 \mathrm{TeV}$ to $40-45 \mathrm{TeV}$ gives

$$
\sqrt{n} \sim \frac{40-45 \mathrm{TeV}}{3 \mathrm{TeV}} \approx 14
$$

So, in $\mathrm{Pb}$-Pb-interactions up to 200 nucleons can be included in the QGM blob (practically half of the total number of interacting nucleons).

Another example of the explanation of an unusual LHC result is an imbalance of jet energies in heavy ion collisions (Fig. 4 [12]). In the decay of top-quark $t \rightarrow$ $W+b$, the kinetic energy is distributed between $T_{b} \approx$ $65 \mathrm{GeV}$ and $T_{W} \approx 25 \mathrm{GeV}$. If we take into account the fly-out energy of $t$-quark, $T_{b}$ will be more than $100 \mathrm{GeV}$. In this case, if $b$-quark gives a jet and $W$ decays into $\sim 20$ pions, the ATLAS experiment's picture can be obtained.

In general, it is possible to show that the proposed model well describes all cosmic ray and collider experiment "unusual" data. 


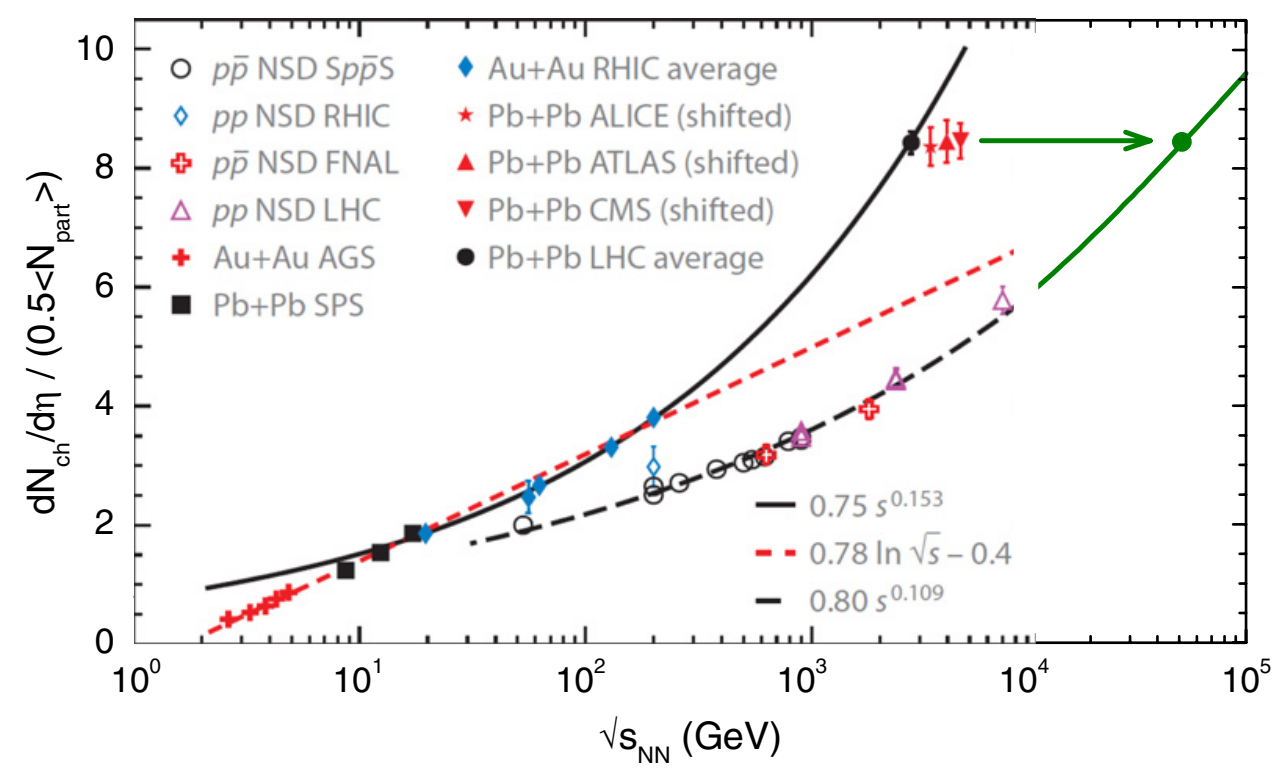

Figure 3. Multiplicity of charged particles in $\mathrm{Pb}-\mathrm{Pb}$-interactions at LHC energy about $3 \mathrm{TeV}$ [11]. Possible shifting of experimental point due to a change of the target mass is shown.
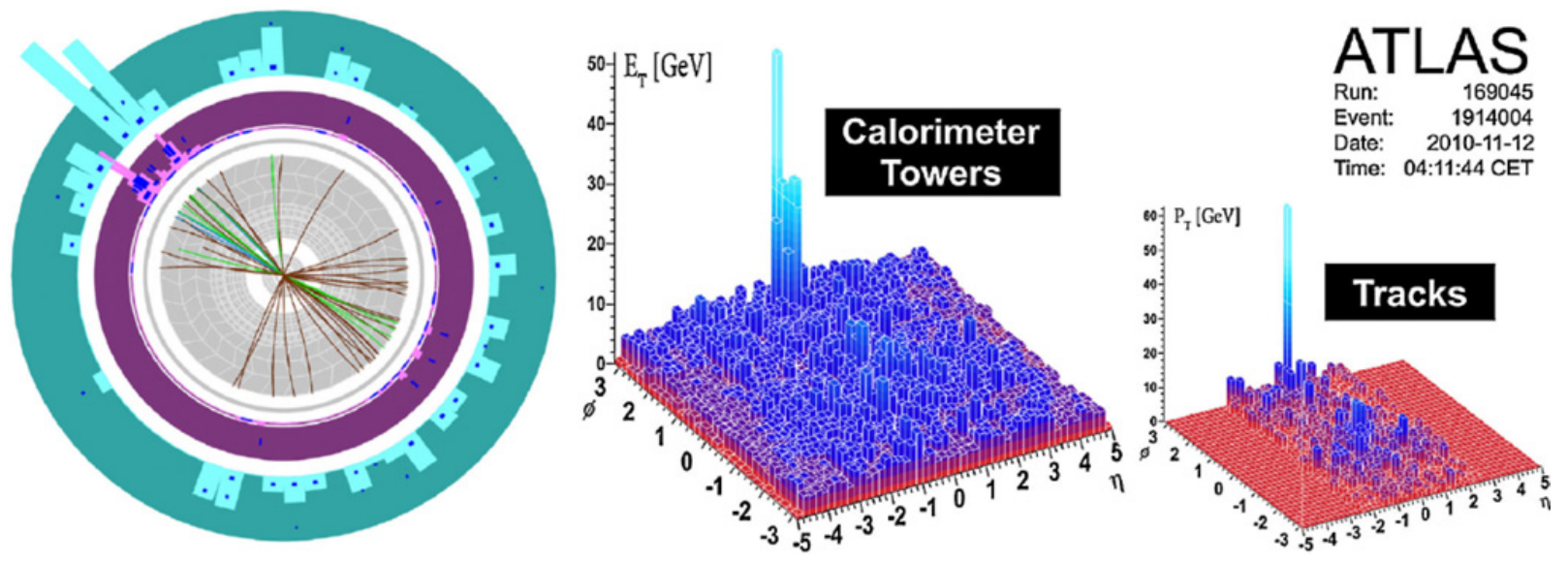

Figure 4. Highly asymmetric dijet event in the ATLAS detector for $\mathrm{Pb}-\mathrm{Pb}$ collisions [12].

\section{What is required to finally check the new model and what are NEEDS-2 for LHC experiment?}

Possibilities to check the new approach are in both cosmic ray investigations and in LHC experiments. In cosmic rays, the most promising possibilities are connected with the study of the energy deposit of muon bundles as a function of primary cosmic ray energy and study of inclusive muon energy spectrum above $100 \mathrm{TeV}$. The first experiment began at the experimental complex NEVOD-DECOR [13]. The second investigation can be made using IceCube experimental data. The preliminary results showed some excess of muons with energies higher than $100 \mathrm{TeV}$ [14]. The further advancement into a higher energy region is possible by means of pair-meter technique [15], and this work is planned. Positive results of both investigations can give very good proofs of truth of the considered model.

But of course, taking into account as mentioned above the skeptic relation of most of the physical community to results of cosmic ray experiments, it is very important to obtain corresponding confirmation from LHC experiments. For that, it is possible to formulate the following NEEDS-2: detailed investigation of nucleusnucleus interactions (not proton-proton or proton-nucleus). It is preferable to investigate relatively light nuclei: nitrogen, oxygen (not heavy nuclei: $\mathrm{Pb}$ or $\mathrm{Au}$ ) which correspond to cosmic ray interactions and multiplicity of secondary particles in which is not so large as for heavy nuclei. Possible directions of nucleus-nucleus investigations:

- search of angular anisotropy of secondary particles analog of alignment;

- search of excess of top-quark production;

- measurements of missing energy;

- other possibilities which can appear in the frame of the model of QGM blob production.

\section{Conclusions}

During NEEDS-1 Workshop, the main aim of cosmic ray investigations was to identify the sources of the 
high energy particles. For that it was necessary to understand the physical processes taking place in the atmosphere. Corresponding tasks for LHC experiments were formulated by G. Schatz in Summary of the NEEDS Workshop [3].

The main aim of NEEDS-2 is to pay attention to the direction of investigations at LHC which follow from results of cosmic ray experiments, and corresponding tasks can be formulated as: a detailed study of multiplicity of secondary particles, missing energy, alignment, top quark production, and possibly other phenomena in nucleusnucleus interactions and their dependence on the beam energy.

Many ideas of a new approach were stimulated by the results obtained at the Unique experimental facility NEVOD with financial support of the Ministry of Education and Science (project RFMEFI59114X0002) and the grant of the President of the Russian Federation (NSh-4930.2014.2). The author thanks Alexey Bogdanov and Rostislav Kokoulin for fruitful discussions and for help in preparing this paper.

\section{References}

[1] L.W. Jones, Cosmic-ray observations need accelerator data, CERN Courier, July/August 2002

[2] NEEDS Workshop discussion, Nucl. Phys. B (Proc. Suppl), 122, 431 (2003)
[3] G. Schatz, Nucl. Phys. B (Proc. Suppl), 122, 462 (2003)

[4] G.B. Khristiansen and G.V. Kulikov, Zhurn. Experim. Teor. Fiz. (in Russian), 35, 635 (1958)

[5] A.A. Petrukhin, Phys. of Atom. Nucl., 66, 517 (2003)

[6] A.A. Petrukhin, Nucl. Phys. B (Proc. Suppl.), 151, 57 (2006)

[7] A.A.Petrukhin, Nucl. Instr. and Meth. in Phys. Res. A, 742, 228 (2014)

[8] A.A. Petrukhin, Nucl. Instr. and Meth. in Phys. Res. A, 692, 228 (2012)

[9] Zuo-Tang Liang and Xin-Nian Weng, Phys. Rev. Lett., 94, 102301 (2005); Phys. Rev. Lett., 96, 039901 (2006)

[10] Jian-Hua Gao et al., Phys. Rev. C, 77, 044902 (2008)

[11] K. Aamodt et al., Phys. Rev. Lett., 105, 252301 (2010)

[12] G. Aad et al., Phys. Rev. Lett., 105, 252303 (2010)

[13] E. A. Kovylyaeva et al., Bull. of the Lebedev Phys. Inst., 41, 218 (2014)

[14] P. Berghaus, C. Xu, Atmospheric muon spectrum from catastrophic energy losses in IceCube, talk presented at 32nd Int. Cosmic Ray Conf., Beijing, China, 2011

[15] R.P. Kokoulin, A.A. Petrukhin, Nucl. Instr. Meth. A, 263, 468 (1988) 